\title{
Skyrmion-Antiskyrmion pair creation by in-plane currents
}

\author{
Martin Stier, ${ }^{1}$ Wolfgang Häusler, ${ }^{1,2}$ Thore Posske, ${ }^{1}$ Gregor Gurski, ${ }^{1}$ and Michael Thorwart ${ }^{1}$ \\ ${ }^{1}$ I. Institut für Theoretische Physik, Universität Hamburg, Jungiusstraße 9, 20355 Hamburg, Germany \\ ${ }^{2}$ Institut für Physik, Universität Augsburg, 86135 Augsburg, Germany
}

\begin{abstract}
Magnetic skyrmions can be considered as topologically protected localized vortex-like spin textures. Due to their stability, their small size, and the possibility to move them by low electric currents they are promising candidates for spintronic devices. Without violating topological protection, it is possible to create skyrmionantiskyrmion pairs, as long as the total charge remains unchanged. We derive a skyrmion equation of motion which reveals how spin-polarized charge currents create skyrmion-antiskyrmion pairs. It allows to identify general prerequisites for the pair creation process. We corroborate these general principles by numerical simulations. On a lattice, where topological protection becomes imperfect, the antiskyrmion partner of the pairs is annihilated and only the skyrmion survives. This eventually changes the total skyrmion number and yields a new way of creating and controlling skyrmions.
\end{abstract}

Magnetic skyrmions (Sks) are vortex-like localized magnetization configurations [1,2] which have been predicted [3] before they were discovered experimentally [4-7] in magnetic layers with a strong spin-orbit interaction [8-10]. Despite their potentially small size $[11,12]$, their thermodynamic stability is considerably strong $[5,7,13]$. This is a consequence of the particular magnetic configuration which can be characterized by a total topological charge or Sk number $Q$. It can take integer values only and therefore cannot be changed continuously $[14,15]$. This feature protects magnetic Sks against typical drawbacks of solid state systems such as disorder or imperfect fabrication $[8,9,16]$. Together with the property of easy repositioning by rather tiny in-plane electrical currents [17-21], this makes single Sks attractive candidates for future racetrack memory devices [17, 22-26]. Creation of Sks has been reported in the vicinity of notches [18], by circular currents [27], by geometrical constraints [28] or by sweeping the external magnetic field [29]. Controlled creation and annihilation of individual Sks has been demonstrated [30]. Each of these processes has to overcome topological protection and the precise mechanism of each way of Sk creation has to be determined.

In this work, we derive a Sk equation of motion which reveals the details of the process how total the topological charge $Q$ changes by an applied in-plane current. We find this to happen in two steps. First, a skyrmion-antiskyrmion (Sk-ASk) pair is created [29] from small spatial fluctuations of the magnetization. Pair creation does not change the total topological charge $Q$, since the Sk and the ASk have equal topological charge of opposite sign, respectively. By the externally applied current the Sk and ASk get spatially further separated. The Sk equation of motion reveals the relevant terms at work which are not captured by the common Thiele approximation [31, 32]. Finally, the ASk, being no stable solution for a given Zeeman field and a Dzyaloshinsky-Moriya interaction, decays due to Gilbert damping. It is this second step, which is ultimately responsible for changing $Q$, crucially relying on dissipation. All general findings are confirmed by extended numerical simulations.

Recently, Sk-ASk pair creation by in-plane currents in systems without Dzyaloshinsky-Moriya interaction has also been reported and investigated numerically [33]. Additionally, Sk creation by in-plane currents also has been observed in experiment [34].

The two-dimensional magnetization configuration $\mathbf{M}(x, y, t)$ of a single current-driven Sk evolves in time according to the extended Landau-Lifshitz-Gilbert (LLG) equation [35-38]

$$
\begin{aligned}
\partial_{t} \mathbf{n}= & -\mathbf{n} \times \mathbf{B}_{\mathrm{eff}}+\alpha \mathbf{n} \times \partial_{t} \mathbf{n} \\
& +\left(\mathbf{v}_{s} \cdot \nabla\right) \mathbf{n}-\beta \mathbf{n} \times\left(\mathbf{v}_{s} \cdot \nabla\right) \mathbf{n}
\end{aligned}
$$

where $\mathbf{n}=\mathbf{M} /|\mathbf{M}|$ is a normalized vector field. All interactions of the Hamiltonian $H$ describing the system are contained in the effective field $\mathbf{B}_{\text {eff }}=-\partial H / \partial \mathbf{n}$. Below, in Eq. (8), we specify the Hamiltonian for a lattice model, but its detailed form is not relevant for the following consideration. $\mathbf{B}_{\mathrm{eff}}$ already contains the gyromagnetic ratio and we set $\hbar=1$. Further important parameters are the Gilbert damping constant $\alpha$ and the non-adiabaticity parameter $\beta$. In this work, we focus specifically on the impact of spin-polarized electric currents $\mathbf{v}_{s}=p a^{3} \mathbf{j}_{c} /(2 e)$ [39] flowing in the magnetic plane with spin polarization $p$ and lattice constant $a$, proportional to a charge current density $\mathbf{j}_{c}$. With the vector field $\mathbf{n}(x, y, t)$, we then define the topological charge density

$$
q(x, y, t)=\mathbf{n} \cdot\left[(\hat{\mathbf{v}} \cdot \nabla) \mathbf{n} \times\left(\hat{\mathbf{v}}_{\perp} \cdot \nabla\right) \mathbf{n}\right],
$$

and the total topological charge

$$
Q=Q(t)=\frac{1}{4 \pi} \int \mathrm{d}^{2} \mathbf{r} q(x, y, t), \quad Q \in \mathbb{Z} .
$$

In fact, this homotopy invariant completely determines the topological properties of Sks even though it does not specify, e.g, the vorticity of a Sk (ASk) without further definitions $[14,15,40]$. In our work, however, the magnetic background will be fixed in such a way that $Q>0(Q<0)$ refers to skyrmions (antiskyrmions) [41]. For convenience, we take the direction of the spin current $\mathbf{v}_{s}$ as reference direction, $\hat{\mathbf{v}}=\mathbf{v}_{s} /\left|\mathbf{v}_{s}\right|$ and $\hat{\mathbf{v}}_{\perp}=\hat{\mathbf{z}} \times \hat{\mathbf{v}}$. While the topological invariant $Q$ is conserved in time at low energies, the time evolution of $q(x, y, t)$ describes the current-induced local motion of Sks. In 
particular, as discussed below, it also describes the generation or annihilation of Sk-ASk pairs.

To reveal the Sk-ASk pair creation mechanism, we decompose the effective field according to

$$
\mathbf{B}_{\mathrm{eff}}=b_{/ /} \mathbf{n}+b_{\perp 1}\left(\mathbf{v}_{s} \cdot \nabla\right) \mathbf{n}+b_{\perp 2} \mathbf{n} \times\left(\mathbf{v}_{s} \cdot \nabla\right) \mathbf{n} .
$$

By combining Eqs. (1), (2) and (4), we readily obtain the Sk equation of motion

$$
\partial_{t} q=-\nabla \cdot\left(\mathbf{j}_{\mathrm{Sk}}^{(1)}+\mathbf{j}_{\mathrm{Sk}}^{(2)}\right),
$$

with the Sk currents

$$
\begin{aligned}
\mathbf{j}_{\mathrm{Sk}}^{(1)} & =-j_{1} q \mathbf{v}_{s}, \\
\mathbf{j}_{\mathrm{Sk}}^{(2)} & =j_{2}\left\{\left[(\hat{\mathbf{v}} \cdot \nabla) \mathbf{n} \cdot\left(\hat{\mathbf{v}}_{\perp} \cdot \nabla\right) \mathbf{n}\right] \mathbf{v}_{s}\right. \\
& \left.\quad-[(\hat{\mathbf{v}} \cdot \nabla) \mathbf{n}]^{2} \mathbf{v}_{\perp}\right\},
\end{aligned}
$$

with contributions parallel to the current flow $\left(\propto \mathbf{v}_{s}\right)$ and perpendicular to it $\left(\propto \mathbf{v}_{\perp} \equiv \hat{\mathbf{z}} \times \mathbf{v}_{s}\right)$. The coefficients read

$$
\begin{aligned}
& j_{1}=\left[1+\alpha \beta+\alpha b_{\perp 1}+b_{\perp 2}\right] /\left(1+\alpha^{2}\right), \\
& j_{2}=\left[\alpha-\beta-b_{\perp 1}+\alpha b_{\perp 2}\right] /\left(1+\alpha^{2}\right) .
\end{aligned}
$$

The Sk equation of motion (5) resembles a continuity equation [42] which connects the topological charge density $q$ with the Sk current density. We note, however, that conservation of $Q$ in Eq. (3) in the present case is not a consequence of Noether's theorem, albeit conserved quantities may still exist for Eq. (5) [43] under continuous variation of $\mathbf{n}$ [26].

The physical meaning of $\mathbf{j}_{\mathrm{Sk}}^{(1)}$ and $\mathbf{j}_{\mathrm{Sk}}^{(2)}$ becomes apparent when we consider Sks in the steady state where $\partial_{t} \mathbf{n}=0$ and thus $\partial_{t} q=0$. For not too large current densities, no major structural changes of the magnetization occur and $\mathbf{B}_{\text {eff }}$ remains parallel to $\mathbf{n}$. Then, the perpendicular components $b_{\perp 1}=b_{\perp 2}$ vanish and the coefficients $j_{1}$ and $j_{2}$ in Eq. (7) are constant. A case of special importance occurs when $\alpha=\beta$, which implies that $j_{1}=1, j_{2}=0$. Then, $\mathbf{j}_{\mathrm{Sk}}^{(2)}=0$, such that the undistorted topological charge density $q$ moves with the velocity $-\mathbf{v}_{s}$, according to Eq. (6a). This motivates us to call $\mathbf{j}_{\mathrm{Sk}}^{(1)}$ a Sk current density. When $\alpha \neq \beta$ (but still assuming $\left.b_{\perp 1}=b_{\perp 2}=0\right), j_{2}$ becomes nonzero. Then, we may rewrite

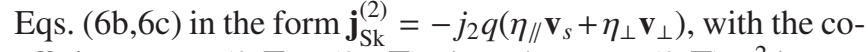
efficients $\eta_{/ /}=(\hat{\mathbf{v}} \cdot \nabla) \mathbf{n} \cdot\left(\hat{\mathbf{v}}_{\perp} \cdot \nabla\right) \mathbf{n} / q$ and $\eta_{\perp}=-[(\hat{\mathbf{v}} \cdot \nabla) \mathbf{n}]^{2} / q$. We can simplify $\eta_{/ /}=\cot \gamma$, where $\gamma$ is the angle between $(\hat{\mathbf{v}} \cdot \nabla) \mathbf{n}$ and $\left(\hat{\mathbf{v}}_{\perp} \cdot \nabla\right) \mathbf{n}$. This term $\propto \eta_{/ /}$only adds to the contribution of $\mathbf{j}_{\mathrm{Sk}}^{(1)}$ (though with a dependence on the shape of the vector field $\mathbf{n}$ ) to drive positive and negative topological charge density along $\pm \mathbf{v}_{s}$ depending on the explicit angle $\gamma$.

Crucial for the following is the term $\propto \eta_{\perp}$. First, it points perpendicularly to the externally applied spin current and, second, it drives negative and positive topological charge densities in opposite directions, as it changes sign under the inversion $q \rightarrow-q$. This is essentially the Sk Hall effect $[14,26,44-$ 47], but for arbitrary topological charge density. We therefore identify the contribution (6c) as being responsible for separating negative from positive topological charge density resulting in a common Sk-ASk pair. The separation takes place perpendicularly to the external current. Actually, this process can be expected to be a common scenario in real materials for sufficiently strong applied charge current densities. The only further prerequisites are $\alpha-\beta \neq 0$ and small spatial fluctuations of the Sk density $q(x, y, t)$, which also imply finite gradients $(\hat{\mathbf{v}} \cdot \nabla) \mathbf{n}$ and $\left(\hat{\mathbf{v}}_{\perp} \cdot \nabla\right) \mathbf{n}$ and thus a finite $\mathbf{j}_{\mathrm{Sk}}^{(2)}$. A finite gradient $\left(\hat{\mathbf{v}}_{\perp} \cdot \nabla\right) \mathbf{n}$ is, strictly speaking, not necessary for a non-vanishing current $\mathbf{j}_{\mathrm{Sk}}^{(2)}$ [cf. Eq. (6c)]. Nevertheless, it is important for a finite divergence $\nabla \cdot \mathbf{j}_{\mathrm{Sk}}^{(2)} \neq 0$. Only in this case, the skyrmion current cannot be gauged away and is physically relevant. Then, regions of opposite signs appear quite naturally in the topologically trivial state $Q=0$, as regions of finite $q$, which we have postulated, have to cancel each other to sum up to zero. Ultimately, a Sk-ASk pair is formed out of these fluctuations. We note in passing that the detailed motion of ASks is typically more complicated than that of Sks, since commonly, an isolated ASk is not a stationary solution and thus, already for $\mathbf{v}_{s}=0, \mathbf{B}_{\text {eff }}$ is clearly not parallel to $\mathbf{n}$, which implies that $b_{\perp} \neq 0$.

In the following, we illustrate these general principles for a concrete model realized by the Hamiltonian [19]

$$
\begin{aligned}
H= & -J \sum_{\mathbf{r}} \mathbf{n}_{\mathbf{r}} \cdot\left(\mathbf{n}_{\mathbf{r}+\mathbf{e}_{x}}+\mathbf{n}_{\mathbf{r}+\mathbf{e}_{y}}\right)-\sum_{\mathbf{r}} \mathbf{B}_{\mathbf{r}} \cdot \mathbf{n}_{\mathbf{r}} \\
& -D \sum_{\mathbf{r}}\left[\left(\mathbf{n}_{\mathbf{r}} \times \mathbf{n}_{\mathbf{r}+\mathbf{e}_{x}}\right) \cdot \mathbf{e}_{x}+\left(\mathbf{n}_{\mathbf{r}} \times \mathbf{n}_{\mathbf{r}+\mathbf{e}_{y}}\right) \cdot \mathbf{e}_{y}\right]
\end{aligned}
$$

defined on lattice sites $\mathbf{r}$ (unit lattice constant) in two dimensions. It supports Sks in a certain parameter regime of the phase diagram. $J$ is the exchange interaction and $D$ the Dzyaloshinsky-Moriya interaction (DMI) strength. We use the values $J=1 \mathrm{meV}, D / J=0.18$ reported for MnSi [19]. Here, we only discuss a bulk DMI which stabilizes Bloch Sks. Yet, we have also verified our findings for systems with an interfacial DMI which stabilizes Néel Sks [48, 49]. No qualitative modifications occur and our findings apply to both kinds of Sks. In the numerical simulations, we use a $L_{x} \times L_{y}=160 \times 160$ square lattice with periodic boundary conditions. For convenience, we translate $v_{s}=p a^{3} I_{c} / 2 e$ into a charge current density $I_{c}$ by assuming full polarization $p=1$ and a lattice constant $a=0.5 \mathrm{~nm}$. Depending on the magnitude of the externally controlled Zeeman field B, either a helical phase, a Sk lattice or the ferromagnetic (field polarized) phase is the ground state [19, 29]. A field $\mathbf{B}=\left(0,0, B_{z}\right)=-0.03 J \hat{\mathbf{z}}$ is in fact strong enough to align all magnetic moments, $\mathbf{n}(x, y, t) \equiv-\hat{\mathbf{z}}$. Then, $q(x, y, t)$ remains zero everywhere and, according to Eqs. (5) and (6), for all times, since $\mathbf{j}_{\mathrm{Sk}}^{(1,2)}=0$, even at non-zero applied current densities.

To realize at least a small initial non-zero topological charge density $q$, we add a tiny modulation to the magnetic field pointing in the $y$-direction, i.e., $B_{y}=b_{0}\left[\sin \left(2 \pi x / L_{x}\right)+\right.$ $\left.\sin \left(2 \pi y / L_{y}\right)\right]$ and $b_{0}=B_{z} / 100$. As a matter of fact, the precise 

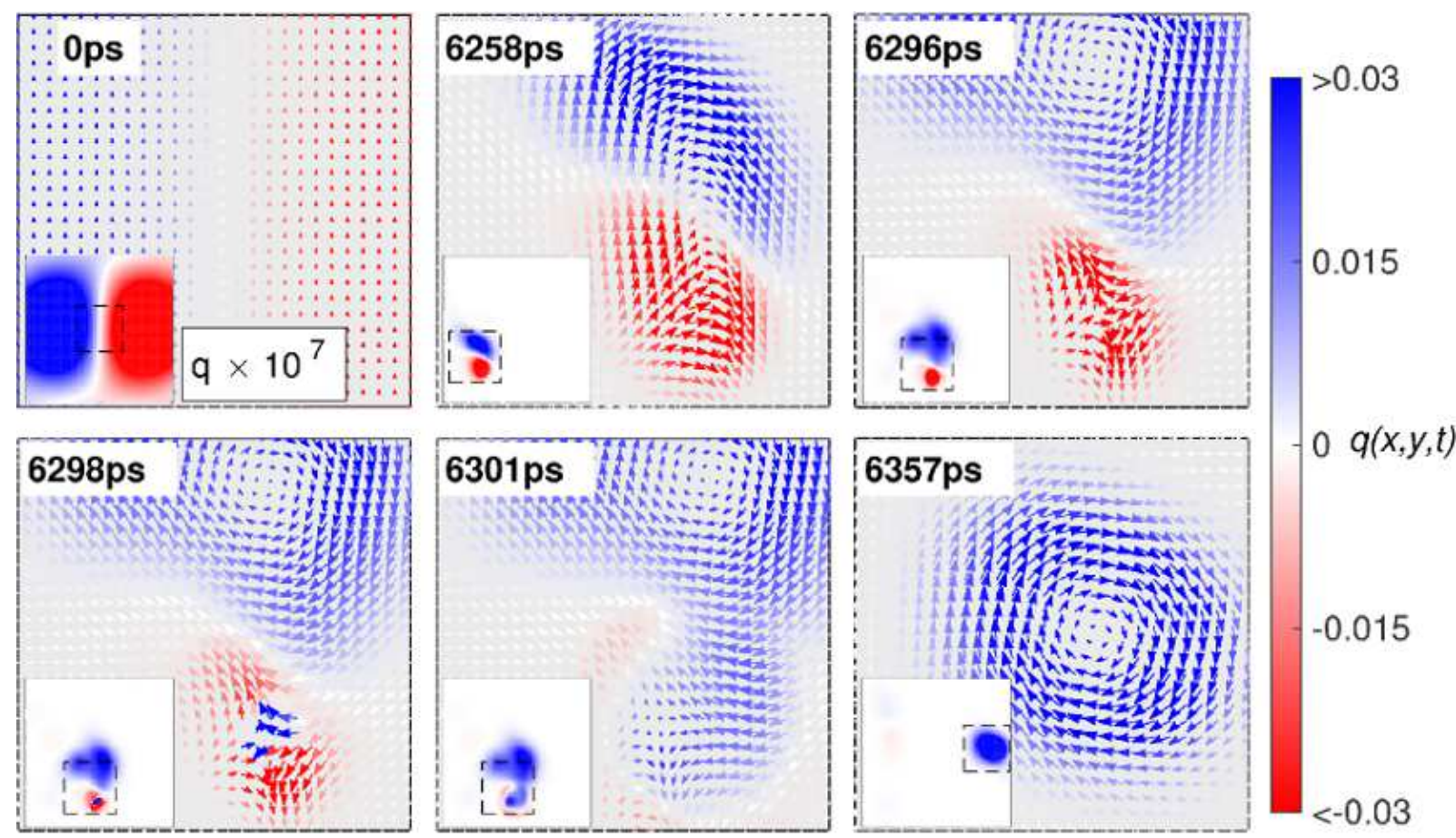

FIG. 1. (Color online). Snapshots of the topological charge density $q$ (insets) in the relevant $80 \times 80$ section of the total lattice and the magnetic texture (arrows) in a magnified section (marked by the dashed rectangles within the insets) at the times as indicated. The initial topological charge adopted by the applied inhomogeneous magnetic field is tiny (see $q$ for $t=0$ which is multiplied by $10^{7}$ for clarity). From these fluctuations the Sk-ASk pair is created by a current in x-direction by separating positive and negative topological charge density perpendicular to the current direction (here, in the $y$-direction). The ASk is eventually destroyed around $t \approx 6300 \mathrm{ps}$ and only the Sk survives. Parameters are chosen as $I_{c}=7.7 \times 10^{11} \mathrm{~A} / \mathrm{m}^{2}, \alpha=0.25$ and $\beta=0$. Color code refers to topological charge density $q$ always.

form of the initial inhomogeneous magnetization configuration is of minor importance. The time evolution of the system is calculated by solving the extended LLG Eq. (1) by standard advanced numerical methods.

Starting from the fully field polarized state $\mathbf{n}(x, y) \equiv-\hat{\mathbf{z}}$, we first let the system accommodate to the additional $B_{y}$ field, at zero external current. After this initial equilibration, we switch on the current at $t=0$ and calculate $q(x, y, t)$ at every time step. A movie of this evolution is available in the SM [50] while a selection of snapshots of $q$ is shown in Fig. 1. Initially, the very small amplitude $b_{0}$ of $B_{y}$ generates a tiny seed topological charge density of both positive and negative sign with an overall $Q=0$. Gradually, under the influence of the external current, Sk-ASk pairs begin to form with growing magnitudes of $q$. Consistent with our theoretical prediction, the Sk and ASk centers separate in the $y$-direction, perpendicular to the external current flow. After its full development, since it is unstable, the ASk disappears on a time scale $\propto 1 / \alpha$. Thereby its diameter shrinks relatively quickly, eventually below the lattice constant. At this moment, $Q(t)$ abruptly changes by one.

As the evolution of Sk-ASk pairs is interfered by the relatively short life time of the ASk we further illustrate the details of this process by an additional movie [50] where we set the DMI to zero. Then, neither the Sk nor the ASk is energetically preferred and the full Sk-ASk pair evolves in time as recently reported in Ref. [33].

The scenario of Sk creation is demonstrated further in Fig. 2, where we show the time-dependence of $Q(t)$. Over large time spans, the total topological charge takes an integer values, while occasionally $Q(t)$ jumps to the next integer within a short transition time. These transitions are accompanied by sudden rises of the total negative topological charge $Q_{\text {ASk }}(t)=\frac{1}{4 \pi} \int_{q<0} \mathrm{~d} x \mathrm{~d} y q(x, y, t)$, a quantity that we define by integrating over negative Sk-density only. During the times when $Q(t)$ stays integer, $Q_{\mathrm{ASk}}(t)$ may decrease gradually with time. This indicates the gradual creation of Sk-ASk pairs, their growth and their spatial separation, before the finally isolated, but unstable ASk annihilates during a time much shorter than the duration of its creation, as described above. This initial gradual development of the first Sk-ASk pair due to a weak spatial inhomogeneity of the Zeeman field is clearly seen in Fig. 2. On the other hand, as soon as a finite number of Sks exist (after 6300 ps in Fig. 2), their intrinsic inhomogeneous magnetization suffices to facilitate further creation of Sk-ASk pairs in their surroundings, even at a homogeneous Zeeman-field as we have convinced ourselves independently.

Since the system starts very close to the ferromagnetic ground state, the Sk creation costs energy. This energy is pumped into the system by the charge current. Figure 2 confirms the connection between the increase of the energy and 


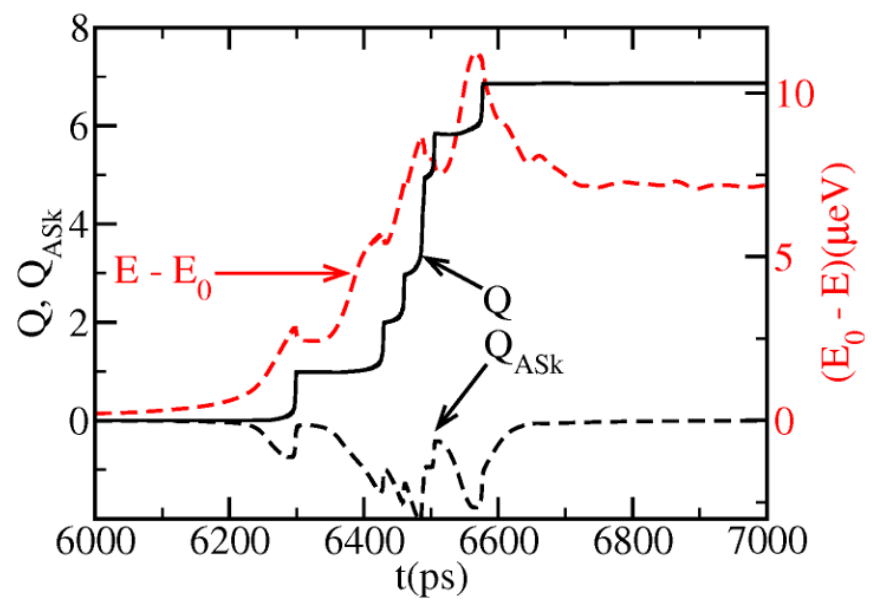

FIG. 2. (Color online). Black solid line: Time-dependence of the total topological charge $Q(t)$. Black dashed line: Time-dependence of the total negative charge defined as $Q_{\mathrm{ASk}}(t)=\frac{1}{4 \pi} \int_{q<0} \mathrm{~d} x \mathrm{~d} y q(x, y, t)$ stemming from negative $q$ only. Note that $Q_{\text {ASk }}$ does not need to be integer and that the restriction to lattice points imposes some small, unimportant ambiguity on the precise determination of $q(x, y, t)$. Red dashed line: Time-dependence of the energy in reference to the initial energy, $E-E_{0} \equiv E(t)-E(t=0)$, per lattice site. Before every $Q$-jump, $Q_{\text {Ask }}$ gradually decreases, accompanied by an increase of the energy which eventually is taken from the external current. Parameters as in Fig. 1.

of negative Sk-density.

The duration for Sk creation can be quantified by the time $\tau$ which we define as the time span from the onset of the current flow till the creation of the first Sk. This creation time is a combination of the time $\tau_{\text {pair }}$ needed to form a sufficiently large Sk-ASk pair and the annihilation time $\tau_{\mathrm{ASk}}$ of the ASk. Since both processes happen at least partially simultaneously, the resulting $\tau$ is not a direct sum of both. Still, $\tau_{\mathrm{ASk}} \ll \tau_{\text {pair }}$ such that we can safely take $\tau \approx \tau_{\text {pair }}$. Since we attribute the creation of Sk-ASk pairs to the existence of a finite $\mathbf{j}_{\mathrm{Sk}}^{(2)}$, we expect Sks to be created faster when the magnitude of $\mathbf{j}_{\mathrm{Sk}}^{(2)}$ is larger. From Eqs. (6b, 6c, 7b) we find $\left|\mathbf{j}_{\mathrm{Sk}}^{(2)}\right| \propto(\alpha-\beta) I_{c}$ in the limit of vanishing $b_{\perp 1}$ and $b_{\perp 2}$. In Fig. 3, this relation between $\tau$ and $\mathbf{j}_{\mathrm{Sk}}^{(2)}$ is confirmed by the numerical results. Indeed, $\tau$ depends on $|\alpha-\beta|$ and $I_{c}$. In particular, no Sks can be created when $\alpha=\beta$ which implies that the dissipative current is essential for the charge current-induced Sk creation. Still, finite creation times appear in an experimentally relevant parameter regime. Finally, we note that even though we have chosen a particular seed magnetic field $B_{y, \mathbf{r}}$ to create topological charge density fluctuations, their precise origin is not important. In fact, only an inhomogeneous $q(x, y)$, besides $\alpha \neq \beta$ and $I_{c} \neq 0$, is necessary for $\mathbf{j}_{\mathrm{Sk}}^{(2)}$ to become non-vanishing. Thus, a multitude of ways are eligible to create such fluctuations, for example by local fields, material modification, or by temperature. On the other hand, a change of $Q$ will often be undesirable in distinct set-ups. Then, $\mathbf{j}_{\mathrm{Sk}}^{(2)}$-contributions to Eqs. (6) should be suppressed by a proper choice of the material with a small $|\alpha-\beta|$, or by avoiding magnetization fluctuations, apart from simply working in the low current regime.

In this work, we have established the skyrmion equation of motion by combining the general definition of the skyrmion density and the extended Landau-Lifshitz-Gilbert equation. We here define skyrmion current densities that conserve the total topological charge of a sample. In the presence of an in-plane spin current, we identify terms that give rise to simple movement of skyrmions against the externally applied charge flow. Other contributions to skyrmion current densities that we identify explicitly drive the separation of positive skyrmion density from negative antiskyrmion density perpendicularly to the charge current flow. These latter contributions eventually cause the creation of skyrmion-antiskyrmion pairs, already out of very small magnetic inhomogeneities. The theoretical predictions are corroborated by numerical simulations and applied to systems with bulk and interfacial DMI.

We acknowledge support from the DFG SFB 668 (project B16).

\section{Detailed spin structure of skyrmionic objects}

In the main body of the paper, we focus on the dynamics of the skyrmion density as the important quantity to describe skyrmions or antiskyrmions, respectively. Still, these objects have an internal magnetic structure given by the magnetization $\mathbf{n}(x, y, t)$. In particular, different kinds of DzyaloshinskiiMoriya interactions (DMI) may stabilize different types of skyrmions. In this Appendix, we show exemplary magnetic structures for

- bulk DMI given by the Hamiltonian $H_{\mathrm{bDMI}}=$ $-D \sum_{\mathbf{r}}\left[\left(\mathbf{n}_{\mathbf{r}} \times \mathbf{n}_{\mathbf{r}+\mathbf{e}_{x}}\right) \cdot \mathbf{e}_{x}+\left(\mathbf{n}_{\mathbf{r}} \times \mathbf{n}_{\mathbf{r}+\mathbf{e}_{y}}\right) \cdot \mathbf{e}_{y}\right]$ which stabilizes Bloch-like skyrmions (cf. Fig. 4 and movie "SK_density_vs_time_bulkDMI . avi”),

- interfacial DMI given by the Hamiltonian $H_{\mathrm{iDMI}}=$ $-D \sum_{\mathbf{r}}\left[(\hat{\mathbf{z}} \times \hat{\mathbf{x}}) \cdot\left(\mathbf{n}_{\mathbf{r}} \times \mathbf{n}_{\mathbf{r}+\mathbf{e}_{x}}\right)+(\hat{\mathbf{y}} \times \hat{\mathbf{z}}) \cdot\left(\mathbf{n}_{\mathbf{r}} \times \mathbf{n}_{\mathbf{r}+\mathbf{e}_{y}}\right)\right]$ which stabilizes Neél-like skyrmions (cf. Fig. 5 and movie "SK_density_vs_time_interfacialDMI .avi"), and,

- no DMI with means stabilization neither of Blochlike nor of Neél-like skyrmions (cf. Fig. 6 and movie "SK_density_vs_time_zeroDMI . avi").

All figures are snapshots taken from movies which we also provide as Supplemental Material online in the "other formats" option on the article's arXiv page. Even though the explicit magnetic structures differ for the according DMI, no qualitative changes for the pair creation process, as described in the main article, were observed.

We have used the same parameter set as before. The calculations were performed on a $L_{x} \times L_{y}=160 \times 160$ square lattice with periodic boundary conditions, external magnetic 

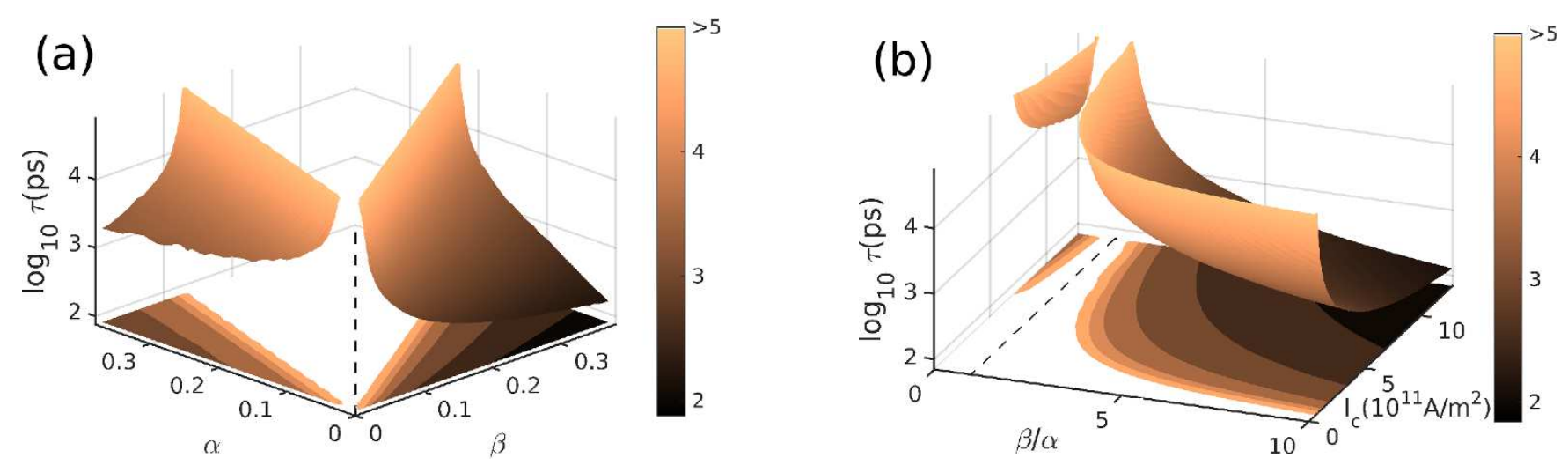

FIG. 3. (Color online). Decadic logarithm of the Sk creation time $\tau$ in dependence of (a) the Gilbert damping constant $\alpha$ and the nonadiabaticity parameter $\beta$ for $I_{c}=10^{12} \mathrm{~A} / \mathrm{m}^{2}$, and, (b) the ratio $\beta / \alpha$ and the charge current density $I_{c}$ for $\alpha=0.05$. Finite creation times are never achieved at $\beta=\alpha$ (dashed lines).

field $\mathbf{B}=\left(0,0, B_{z}\right)=-0.03 J \hat{\mathbf{z}}$, spin velocity $v_{s}=-300 \mathrm{~m} / \mathrm{s}$, Gilbert damping $\alpha=0.25$ and non-adiabaticity $\beta=0$. To create initial fluctuations of the skyrmion density, a tiny modulation to the magnetic field pointing in the $y$-direction, i.e., $B_{y}=b_{0}\left[\sin \left(2 \pi x / L_{x}\right)+\sin \left(2 \pi y / L_{y}\right)\right]$ and $b_{0}=B_{z} / 100$ has been added to the external field.

[1] T. Skyrme, Nucl. Phys. 31, 556 (1962).

[2] N. Kiselev, A. Bogdanov, R. Schäfer, and U. Rößler, J. Phys. D Appl. Phys. 44, 392001 (2011).

[3] A. Bogdanov and A. Hubert, J. Mag. Mag. Mat. 138, 255 (1994).

[4] X. Yu, N. Kanazawa, Y. Onose, K. Kimoto, W. Zhang, S. Ishiwata, Y. Matsui, and Y. Tokura, Nat. Mater. 10, 106 (2011).

[5] S. Heinze, K. Von Bergmann, M. Menzel, J. Brede, A. Kubetzka, R. Wiesendanger, G. Bihlmayer, and S. Blügel, Nat. Phys. 7, 713 (2011).

[6] C. Hanneken, F. Otte, A. Kubetzka, B. Dupé, N. Romming, K. von Bergmann, R. Wiesendanger, and S. Heinze, Nat. nanotechnol. 10, 1039 (2015).

[7] S. Mühlbauer, B. Binz, F. Jonietz, C. Pfleiderer, A. Rosch, A. Neubauer, R. Georgii, and P. Böni, Science 323, 915 (2009).

[8] A. Bogdanov, Sov. Phys. JETP Lett. 62, 247 (1995).

[9] A. Bogdanov and A. Hubert, J. Mag. Mag. Mat. 195, 182 (1999).

[10] U. Rößler, A. Bogdanov, and C. Pfleiderer, Nature 442, 797 (2006).

[11] K. Shibata, X. Yu, T. Hara, D. Morikawa, N. Kanazawa, K. Kimoto, S. Ishiwata, Y. Matsui, and Y. Tokura, Nat. nanotechnol. 8, 723 (2013).

[12] N. Romming, A. Kubetzka, C. Hanneken, K. von Bergmann, and R. Wiesendanger, Phys. Rev. Lett. 114, 177203 (2015).

[13] J. Hagemeister, N. Romming, K. von Bergmann, E. Vedmedenko, and R. Wiesendanger, Nat. Comm. 6, 8455 (2015).

[14] X.-L. Qi, Y.-S. Wu, and S.-C. Zhang, Phys. Rev. B 74, 085308 (2006).

[15] M. W. Hirsch, "Degrees, intersection numbers, and the euler characteristic," in Differential Topology (Springer New York, New York, NY, 1976) pp. 120-141.
[16] A. Rosch, Nat. nanotechnol. 8, 160 (2013).

[17] J. Sampaio, V. Cros, S. Rohart, A. Thiaville, and A. Fert, Nat. nanotechnol. 8, 839 (2013).

[18] J. Iwasaki, M. Mochizuki, and N. Nagaosa, Nat. nanotechnol. 8, 742 (2013).

[19] J. Iwasaki, M. Mochizuki, and N. Nagaosa, Nat. Comm. 4, 1463 (2013).

[20] F. Jonietz, S. Mühlbauer, C. Pfleiderer, A. Neubauer, W. Münzer, A. Bauer, T. Adams, R. Georgii, P. Böni, R. Duine, et al., Science 330, 1648 (2010).

[21] X. Yu, N. Kanazawa, W. Zhang, T. Nagai, T. Hara, K. Kimoto, Y. Matsui, Y. Onose, and Y. Tokura, Nat. Comm. 3, 988 (2012).

[22] S. Krause and R. Wiesendanger, Nat. Mater. 15, 493 (2016).

[23] S. Woo, K. Litzius, B. Krüger, M.-Y. Im, L. Caretta, K. Richter, M. Mann, A. Krone, R. M. Reeve, M. Weigand, et al., Nat. Mater. , 501 (2016).

[24] X. Zhang, G. Zhao, H. Fangohr, J. P. Liu, W. Xia, J. Xia, and F. Morvan, Sci. Rep. 5 (2015).

[25] A. Fert, V. Cros, and J. Sampaio, Nat. nanotechnol. 8, 152 (2013).

[26] N. Nagaosa and Y. Tokura, Nat. nanotechnol. 8, 899 (2013).

[27] Y. Tchoe and J. H. Han, Phys. Rev. B 85, 174416 (2012).

[28] W. Jiang, P. Upadhyaya, W. Zhang, G. Yu, M. B. Jungfleisch, F. Y. Fradin, J. E. Pearson, Y. Tserkovnyak, K. L. Wang, O. Heinonen, S. G. E. te Velthuis, and A. Hoffmann, Science 349, 283 (2015)

[29] W. Koshibae and N. Nagaosa, Nat. Comm. 7 (2016).

[30] N. Romming, C. Hanneken, M. Menzel, J. E. Bickel, B. Wolter, K. von Bergmann, A. Kubetzka, and R. Wiesendanger, Science 341, 636 (2013).

[31] A. Thiele, Phys. Rev. Lett. 30, 230 (1973).

[32] J. Müller and A. Rosch, Phys. Rev. B 91, 054410 (2015).

[33] K. Everschor-Sitte, M. Sitte, T. Valet, J. Sinova, and A. Abanov, arXiv:1610.08313 (2016).

[34] X. Yu, D. Morikawa, Y. Tokunaga, M. Kubota, T. Kurumaji, H. Oike, M. Nakamura, F. Kagawa, Y. Taguchi, T.-h. Arima, M. Kawasaki, and Y. Tokura, Adv. Mat. , 1606178 (2017), 1606178 .

[35] G. Tatara, H. Kohno, and J. Shibata, Phys. Rep. 468, 213 (2008).

[36] Z. Li and S. Zhang, Phys. Rev. Lett. 92, 207203 (2004).

[37] Y. B. Bazaliy, B. Jones, and S.-C. Zhang, Phys. Rev. B 57, R3213 (1998).

[38] M. Lakshmanan, Philosophical Transactions of the Royal Soci- 

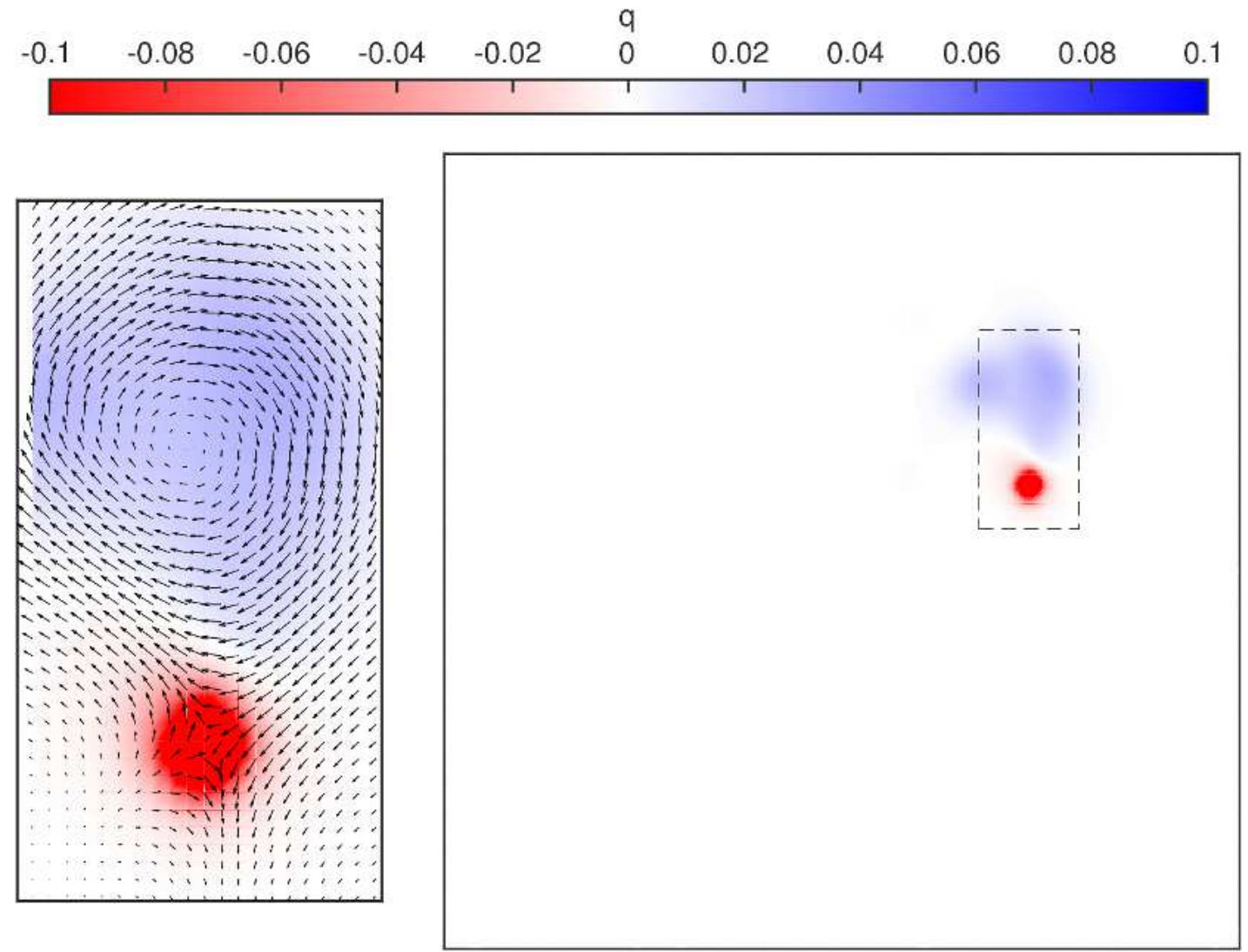

FIG. 4. Skyrmion density $q(x, y, t)$ at $t=6296 \mathrm{ps}$. The marked area is magnified on the left-hand side where the arrows also show the magnetization $n(x, y, t)$ in the $x y$ plane. The bulk Dzyaloshinskii-Moriya interaction $H_{\mathrm{bDMI}}=-D \sum_{\mathbf{r}}\left[\left(\mathbf{n}_{\mathbf{r}} \times \mathbf{n}_{\mathbf{r}+\mathbf{e}_{x}}\right) \cdot \mathbf{e}_{x}+\left(\mathbf{n}_{\mathbf{r}} \times \mathbf{n}_{\mathbf{r}+\mathbf{e}_{y}}\right) \cdot \mathbf{e}_{y}\right]$ stabilizes Bloch-like skyrmions. 


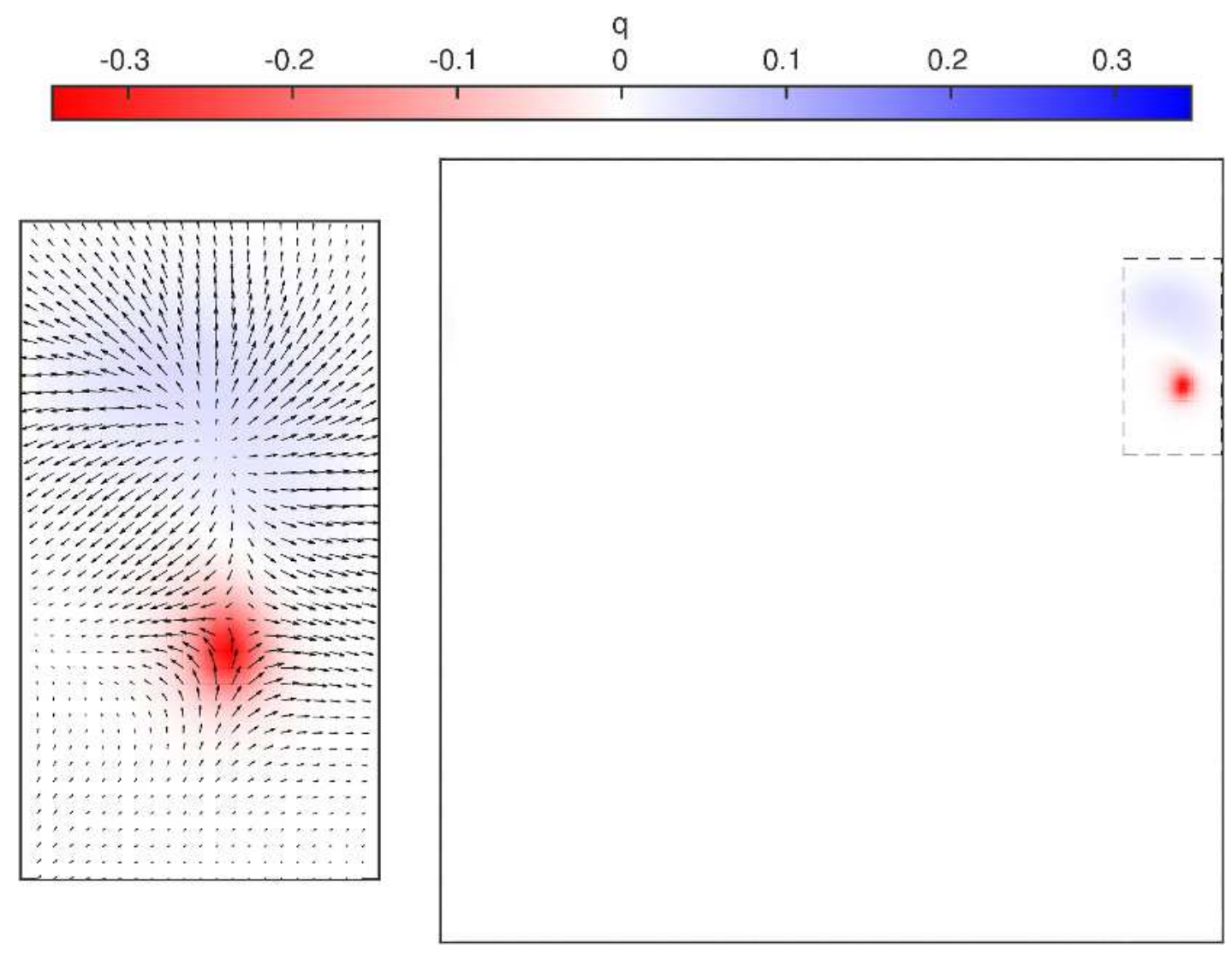

FIG. 5. Skyrmion density $q(x, y, t)$ at $t=4933$ ps. The marked area is magnified on the left-hand side where the arrows also show the magnetization $n(x, y, t)$ in the $x y$ plane. In contrast to Fig. 4 an interfacial Dzyaloshinskii-Moriya interaction $H_{\mathrm{iDMI}}=$ $-D \sum_{\mathbf{r}}\left[(\hat{\mathbf{z}} \times \hat{\mathbf{x}}) \cdot\left(\mathbf{n}_{\mathbf{r}} \times \mathbf{n}_{\mathbf{r}+\mathbf{e}_{x}}\right)+(\hat{\mathbf{y}} \times \hat{\mathbf{z}}) \cdot\left(\mathbf{n}_{\mathbf{r}} \times \mathbf{n}_{\mathbf{r}+\mathbf{e}_{y}}\right)\right]$ has been used, which stabilizes Neél-like skyrmions.

ety of London A: Mathematical, Physical and Engineering Sciences 369, 1280 (2011).

[39] S. Zhang and S. S.-L. Zhang, Phys. Rev. Lett. 102, 086601 (2009).

[40] C. Heo, N. S. Kiselev, A. K. Nandy, S. Blügel, and T. Rasing, Sci. Rep. 6 (2016).

[41] A. Bogdanov and D. Yablonskii, Zh. Eksp. Teor. Fiz 95, 182 (1989).

[42] M. Garst, "Topological skyrmion dynamics in chiral magnets," in Topological Structures in Ferroic Materials: Domain Walls, Vortices and Skyrmions, edited by J. Seidel (Springer International Publishing, Cham, 2016) pp. 29-53.

[43] G. S. Frederico and D. F. Torres, J. Math. Anal. Appl. 334, 834 (2007).

[44] M. Stone, Phys. Rev. B 53, 16573 (1996).
[45] Y. Nakatani, J. Shibata, G. Tatara, H. Kohno, A. Thiaville, and J. Miltat, Phys. Rev. B 77, 014439 (2008).

[46] X. Zhang, G. Yu, W. Zhang, X. Wang, M. Benjamin Jungfleisch, J. E. Pearson, X. Cheng, O. Heinonen, K. L. Wang, Y. Zhou, A. Hoffmann, and S. G. E. te Velthuis, Nat. Phys. 13, 162-169 (2016).

[47] K. Litzius, I. Lemesh, B. Krüger, P. Bassirian, L. Caretta, K. Richter, F. Büttner, K. Sato, O. A. Tretiakov, J. Förster, R. M. Reeve, M. Weigand, I. Bykova, H. Stoll, G. Schutz, G. S. D. Beach, and M. Kläui, Nat. Phys. 13, 170-175 (2016).

[48] A. Thiaville, S. Rohart, É. Jué, V. Cros, and A. Fert, Europhys. Lett. 100, 57002 (2012).

[49] M. Benitez, A. Hrabec, A. Mihai, T. Moore, G. Burnell, D. McGrouther, C. Marrows, and S. McVitie, Nat. Comm. 6 (2015).

[50] See Supplemental Material at the ancillary file section of arXiv ("other formats") for movies of skyrmion-pair evolution. 
(a)

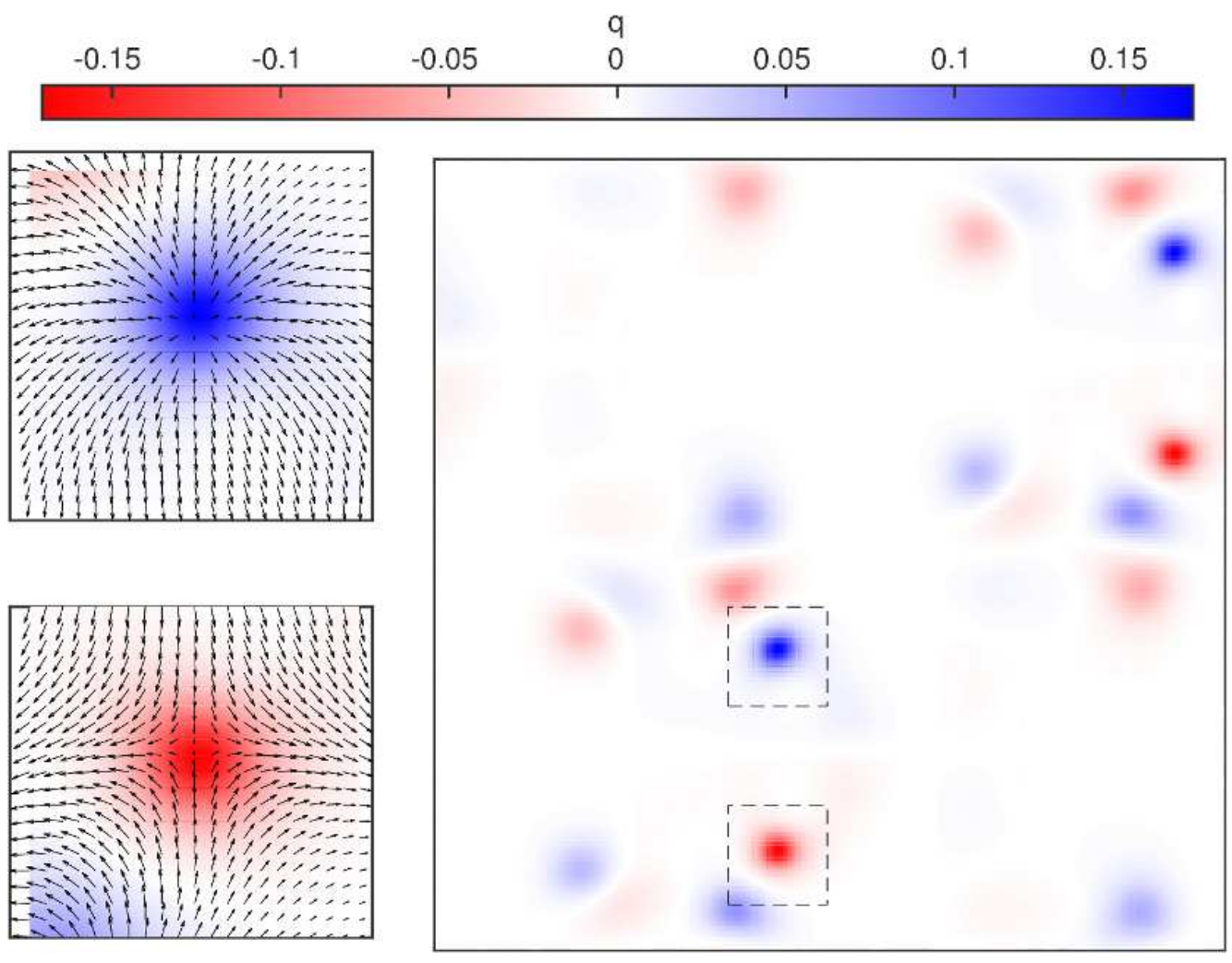

(b)

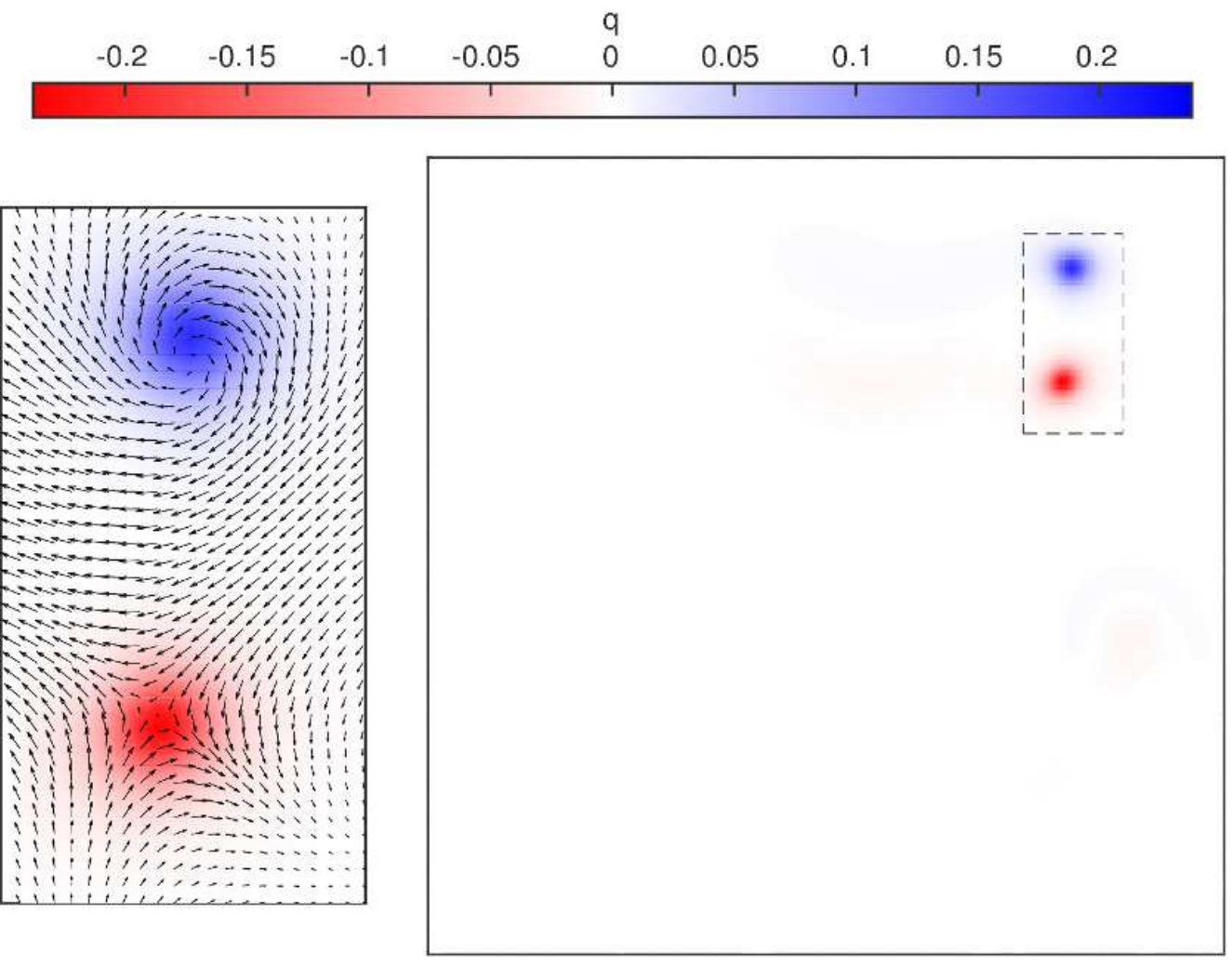

FIG. 6. Skyrmion density $q(x, y, t)$ at (a) $t=10772$ ps and (b) $t=14392$ ps. The marked areas are magnified on the left-hand side where the arrows also show the magnetization $n(x, y, t)$ in the $x y$ plane. The Dzyaloshinskii-Moriya interaction is set to zero. This has two consequences: (i) neither the skyrmion nor the antiskyrmion is preferred and both types are symmetrically created and (ii) skyrmions can be of Neél or Bloch shape. 